\title{
Experimental and theoretical investigations of magnetic anisotropy and magnetic hardening at molecule/ferromagnet interfaces
}

\author{
K. Bairagi, ${ }^{1}$ A. Bellec, ${ }^{1}$ V. Repain,,${ }^{1, *}$ C. Fourmental, ${ }^{1}$ C. Chacon, ${ }^{1}$ Y. Girard,${ }^{1}$ J. Lagoute, ${ }^{1}$ S. Rousset,${ }^{1}$ \\ L. Le Laurent, ${ }^{2}$ A. Smogunov, ${ }^{2}$ and C. Barreteau ${ }^{2}$ \\ ${ }^{1}$ Laboratoire Matériaux et Phénomènes Quantiques, Sorbonne Paris Cité, Université Paris Diderot-Paris 7, UMR CNRS 7162, \\ 10 rue Alice Domon et Léonie Duquet, 75205 Paris Cedex 13, France \\ ${ }^{2}$ SPEC, CEA, CNRS, Université Paris-Saclay, CEA Saclay, 91191 Gif sur Yvette, France
}

(Received 22 January 2018; revised manuscript received 28 June 2018; published 27 August 2018)

\begin{abstract}
We measure by means of in situ magneto-optical Kerr effect the magnetism of ultrathin magnetic films supported on gold and platinum during the capping with molecular layers. We observe both for $\mathrm{C}_{60}$ and $\mathrm{Alq}_{3}$ molecules deposited on Co films an enhancement of the out-of-plane magnetic anisotropy, leading to a magnetic hardening for out-of-plane magnetization. Surprisingly, the amplitude of this hardening depends strongly on the underlying substrate. While we find an increase of the coercive field around $100 \%$ for $\mathrm{Co} / \mathrm{Au}(111)$, it can reach almost $600 \%$ on $\mathrm{Co} / \mathrm{Pt}(111)$. Those results are discussed by using both tight-binding and $a b$ initio calculations. It is shown on the case of $\mathrm{C}_{60}$ that both the adsorption geometry and the strain in the magnetic layer can be key ingredients to predict the quantitative change of magnetic anisotropy induced by the interface between a magnetic material and a molecular layer. On the contrary, the crystalline stacking of the Co has a minor influence.
\end{abstract}

DOI: 10.1103/PhysRevB.98.085432

\section{INTRODUCTION}

Hybrid materials mixing molecular and inorganic ferromagnetic layers have shown in the past decade unexpected spintronic properties. Spin valve structures with $\mathrm{Alq}_{3}$ already showed very high magnetoresistive response and were studied extensively for different device geometries both at low temperature and room temperature [1-3]. Spin valve structure with $\mathrm{C}_{60}$ molecule also showed magnetoresistive effect at room temperature for molecular layers up to $30 \mathrm{~nm}[4,5]$. Generally, the device performance in organic spin valves depends a lot on the nature of the interface between the organic and the ferromagnetic layer [6-8]. Understanding the interaction of molecules with the ferromagnet is therefore needed for determining the spin injection, detection, and overall magnetoresistive behavior of the device [9-12]. But less attention was given on the influence of molecules on the ferromagnetic electrode, especially regarding interfacial perpendicular magnetic anisotropy (PMA) and coercivity [13-15] that both play an important role in realizing efficient spin valve structures. Indeed, interfacial PMA, like the one used nowadays between $\mathrm{Fe}$ and $\mathrm{MgO}$, is required to obtain out-of-plane magnetization devices and magnetic hardening is an important property to obtain the so called magnetic pinned layer, i.e., that does not switch under a moderate applied magnetic field.

It has been already studied that $\pi$ conjugated organic semiconductors interacting with $d$ electrons of ferromagnet give rise to magnetic anisotropy. In case of $\mathrm{Co}$, the interaction gives rise to enhanced PMA with respect to the bare Co surface due to selective orbital hybridization at the interface $[13,16]$. The coercivity of the ferromagnetic layer was observed to increase

\footnotetext{
*vincent.repain@univ-paris-diderot.fr
}

by molecular capping both from theoretical calculations and experimental observations on few cases [15-17]. However, more systematic investigations on model systems are needed to understand in details the interplay between the structure and the magnetic anisotropy at the interface, which is required for a smarter design of molecular spintronic devices.

In this paper we present our investigations on the change in magnetic properties of ultrathin magnetic films [grown either on $\mathrm{Au}(111)$ or $\mathrm{Pt}(111)$ single crystal substrates] upon $\mathrm{C}_{60}$ or $\mathrm{Alq}_{3}$ molecule depositions. In situ depositions of both magnetic materials and molecules enable us to study precisely the molecule/ferromagnet interfaces. Magnetic hardening of Co ultrathin film with $\mathrm{C}_{60}$ molecule and the change in magnetic anisotropy energy (MAE) due to different molecules is also addressed for different substrates. The paper is organized as follows. In Sec. II, we give the technical details of the experiments and of the calculations. In Sec. III, we present and analyze the experimental results. In Sec. IV, we show the results of our calculations and we conclude in Sec. V.

\section{EXPERIMENTAL AND CALCULATION DETAILS}

\section{A. Experimental setups and samples}

The experiments were performed in an ultrahigh vacuum (UHV) setup having a base pressure less than $10^{-10} \mathrm{mbar}$ with separate chambers for sample preparation, magneto optic Kerr effect (MOKE) experiment, and scanning tunneling microscopy (STM) imaging. Both $\mathrm{Au}(111)$ and $\mathrm{Pt}(111)$ substrates were prepared by $\mathrm{Ar}^{+}$ion sputtering at $900 \mathrm{eV}$ and subsequent annealing. The annealing temperature was $750 \mathrm{~K}$ for $\mathrm{Au}(111)$, whereas a flash annealing up to $1000 \mathrm{~K}$ was done for $\mathrm{Pt}(111)$. The cleanness of the substrates (typically less than one per thousand of impurities) were checked by both STM and Auger electron spectroscopy. The evaporation of cobalt was done 
using a standard physical vapor deposition method by electron beam bombardment to a $2 \mathrm{~mm}$ diameter Co rod (EFM 3, Focus $\mathrm{GmbH})$. The evaporation was controlled by a flux monitor at a constant rate of about $0.2 \mathrm{ML} / \mathrm{min}$. The calibration has first been done using STM images of submonolayer Co deposited on $\mathrm{Au}(111)$ and $\operatorname{Pt}(111)$. However, the coverage accuracy is limited by the reproducibility of the flux monitor and the tip convolution in the analysis of STM images. Considering those sources of error, we consider that our calibration of the Co deposition has an error bar of $\pm 15 \%$. In order to get a better precision in the comparison of different samples, we have therefore decided to calibrate our Co thicknesses using the magnetic measurements and more precisely the spin reorientation transition. The critical thickness where the magnetization switches from out of plane to in plane, that we define where the ratio of the remnant magnetization over the saturation magnetization equals 0.5 , has been fixed to $4.2 \mathrm{ML}$ for $\mathrm{Co} / \mathrm{Au}(111)$ and 4.5 ML for Co/Pt(111), in good agreement with STM calibrations and literature $[18,19]$. It is however to be noticed that the precise shape of the hysteresis cycles during the SRT is not perfectly reproducible from sample to sample on $\operatorname{Pt}(111)$, whereas it is on $\mathrm{Au}(111)$. As a matter of fact, we believe that our uncertainty of the Co thickness is higher for the $\mathrm{Co} / \mathrm{Pt}(111)$ system than for the $\mathrm{Co} / \mathrm{Au}(111)$. A homemade Knudsen cell was used for $\mathrm{C}_{60}$ evaporation, whereas $\mathrm{Alq}_{3}$ molecules were evaporated from a commercial Knudsen cell (OLED effusion cell, CreaTec $\mathrm{GmbH}$ ). The flux of evaporation was about $0.01 \mathrm{ML} / \mathrm{min}$ for $\mathrm{C}_{60}$ evaporation and $0.05 \mathrm{ML} / \mathrm{min}$ for $\mathrm{Alq}_{3}$ evaporation. The calibration of the molecule depositions were done further by using STM images of submonolayer depositions on $\mathrm{Au}(111)$. It is only a rough estimate for $\mathrm{Alq}_{3}$ which is difficult to image. Magnetic hysteresis cycles were recorded in situ during material depositions every $20 \mathrm{~s}$ with an external magnetic coil having field sweep rate of $1 \mathrm{~Hz}$ and a maximum field of $68 \mathrm{mT}$ at room temperature and $272 \mathrm{mT}$ when the coil is cooled down at liquid nitrogen temperature. In all cases a polar MOKE configuration was used where the magnetic field was applied parallel to the surface normal to detect the out-of-plane magnetization of the sample. The total magnetic anisotropy energy (MAE) of the samples can be deduced from hard axis magnetometry. This MAE is the sum of the magnetocrystalline anisotropy (MCA) and the shape anisotropy. We define it as the difference of energy between an in-plane and an out-of-plane magnetization, meaning that a positive value favors out-ofplane magnetization. All the cycles shown are raw data except that the Kerr intensity, measured in $\mathrm{mV}$, has been systematically normalized to unity for a saturated cycle.

\section{B. Tight-binding and density functional theory methods}

Calculations based on the density functional theory (DFT) were employed to determine various relaxed structures and, for the sake of comparison, two different $a b$ initio electronic structure software packages have been used: QUANTUM ESPRESSO (QE) [20] based on a plane wave expansion of the wave functions and ATK from QUANTUMWISE [21,22] based on a localized basis set expansion. These calculations were performed within the generalized gradient approximation using the Perdew, Burke, and Ernzerhof parametrization without spin-orbit coupling. For the systems with $4 \times 4$ in plane periodicity (see Secs. IV C and IVD) the number of $k$ points used was $3 \times 3$ in the surface Brillouin zone, which was sufficient to obtain converged structure.

The MCA has been calculated in a magnetic tight-binding (TB) scheme including spin-orbit coupling and using the force theorem (details of the model are described in Ref. [23]). A selfconsistent field (scf) calculation without SOC is first performed followed by a single diagonalization including SOC starting from the scf charge distribution. For the systems with $4 \times 4$ in plane periodicity the number of $k$ points used was $10 \times$ 10 and $25 \times 25$ in the surface Brillouin zone for TB without SOC and with SOC, respectively. The MCA is defined as the difference of energy between an in-plane magnetization and out-of-plane magnetization MCA $=E_{\|}-E_{\perp}$; positive MCA indicates a tendency to out-of-plane magnetization. The total energy difference is then decomposed over the different atomic sites of the system as explained in Ref. [24].

\section{EXPERIMENTAL RESULTS}

\section{A. $\mathrm{Alq}_{3} / \mathrm{Co} / \mathrm{Au}(111)$}

It is well known that at a Co thickness $\left(t_{c}\right)$ of $4.2 \mathrm{ML}$ on $\mathrm{Au}(111)$ a spin reorientation transition (SRT) from out of plane to in plane occurs [18,25]. For a 5.5 ML Co film with in-plane magnetization, a reverse SRT was observed after the adsorption of a $\mathrm{C}_{60}$ molecular overlayer due to an enhanced PMA [16]. In order to better understand the role of the molecular nature and structure on the MAE change of a Co layer, we performed a similar experiment with the $\mathrm{Alq}_{3}$ molecule. $\mathrm{Alq}_{3}$ is a very different molecule from $\mathrm{C}_{60}$, generally showing some amorphous structure in thin films, and particularly important in spintronic studies as it is used in most of the devices studied in the literature. In Fig. 1(a) we demonstrate the change in magnetization of a 5.8 ML of $\mathrm{Co}$ on $\mathrm{Au}(111)$ during the deposition of a monolayer of $\mathrm{Alq}_{3}$ on top of it. It is clear from the raw hysteresis cycles that the magnetization goes from in plane (reversible and unsaturated cycles) to out of plane (irreversible and saturated cycles). Thus $\mathrm{Alq}_{3}$ is inducing a PMA to the underlying Co film in a very similar manner to $\mathrm{C}_{60}$ [16]. The STM image [cf. bottom right inset of Fig. 1(b)] shows the presence of the adsorbed $\mathrm{Alq}_{3}$ molecule on Co. It is worth noting that no ordered structure was observed and the images are blurry, certainly due to the deformation of the molecules under the tip.

To quantify the change in MAE due to the molecular deposition we performed a similar set of experiments where the magnetic cycles remained in plane after a full monolayer deposition of molecules. We then extracted the MAE change from the evolving slope of the cycles using hard axis magnetometry (see the Supplemental Material of Ref. [16] for details). The similar trend in the change of MAE, both qualitatively and quantitatively [cf. Fig. 1(b)], is in good agreement with the interpretation that it comes from the same specific hybridization between molecules and cobalt at the interface. State-of-the-art $a b$ initio calculations in the case of $\mathrm{C}_{60} / \mathrm{Co}$ interface showed that the increase in PMA is mainly resulting from the hybridization of $\mathrm{C} p_{z}$ orbitals in contact with the Co $d_{z^{2}}$ orbitals [16]. The suppression of the $d_{z^{2}}$ negative contribution to the MCA induced by this hybridization process 
(a)

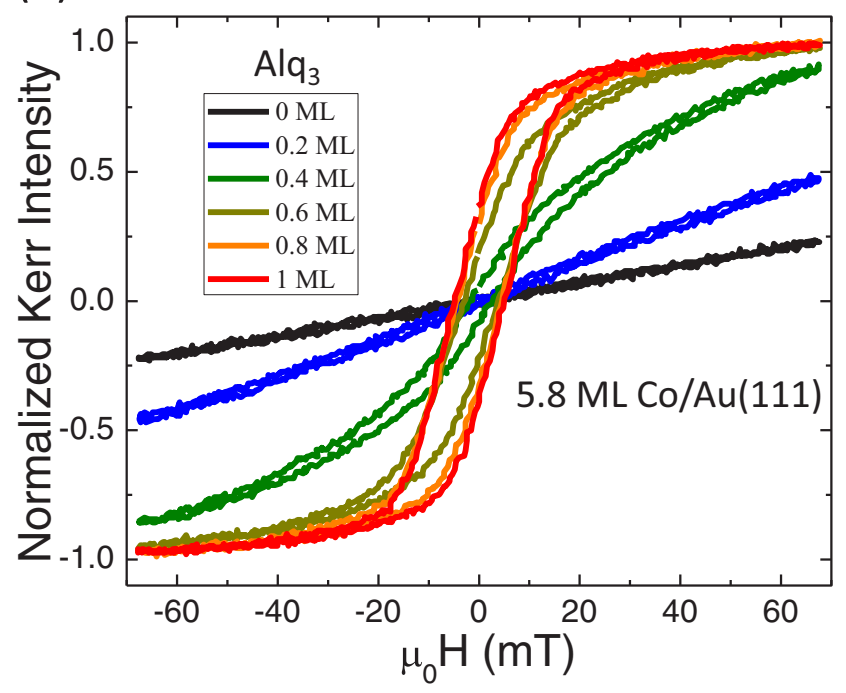

(b)

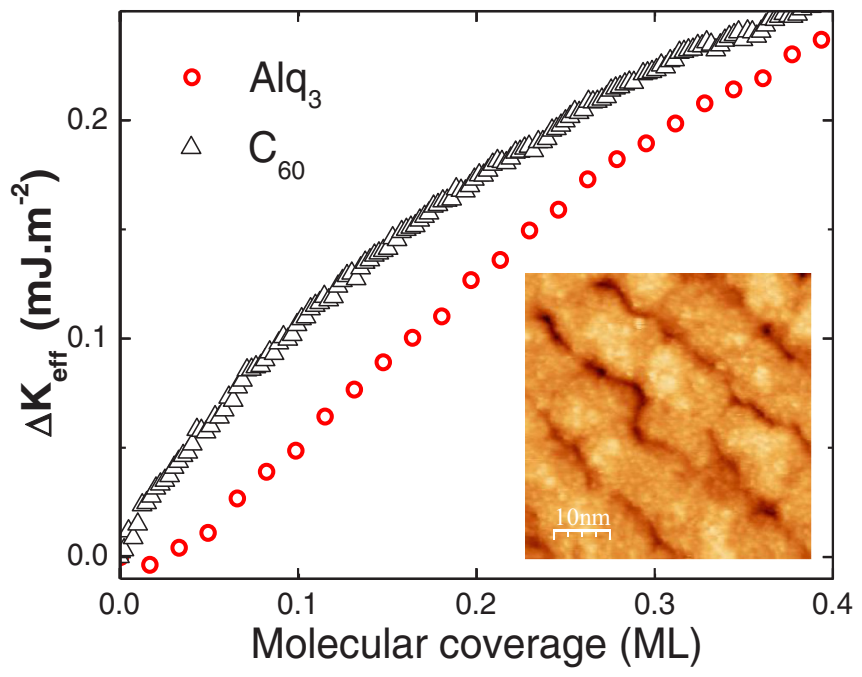

FIG. 1. (a) Raw hysteresis cycles recorded by polar MOKE in situ during growth of $\mathrm{Alq}_{3}$ on 5.8 ML Co/Au(111) for each 0.2 ML increase of $\mathrm{Alq}_{3}$ coverage. (b) Comparison of the change of interfacial magnetic anisotropy for $\mathrm{C}_{60}$ on $6.4 \mathrm{ML} \mathrm{Co} / \mathrm{Au}(111)$ and $\mathrm{Alq}_{3}$ on 6.7 ML Co/Au(111). In inset, $50 \times 50 \mathrm{~nm}^{2} \mathrm{STM}$ image recorded at $2 \mathrm{~V}, 100 \mathrm{pA}$ showing the presence of a disordered layer of $\mathrm{Alq}_{3}$ on $\mathrm{Co} / \mathrm{Au}(111)$.

is believed to be the main reason for the overall increase of MAE towards out-of-plane configuration. In the case of $\mathrm{Alq}_{3}$, it has been shown that a chemical bonding was occurring at the $\mathrm{Alq}_{3} / \mathrm{Co}$ interface $[26,27]$. Ab initio calculations show that the molecule indeed strongly deforms with two quinoline ligands lying flat on the surface, inducing some specific C $p_{z}-$ Co $d_{z^{2}}$ hybridization [28], giving rise to a change of interfacial MAE very similar to $\mathrm{C}_{60}$.

\section{B. $\mathrm{C}_{60} / \mathrm{Co} / \mathrm{Pt}(111)$}

In order to better understand the role of parameters such as strain on the magnetic properties of molecule/Co interfaces we have decided to perform several experiments on Co films grown on $\mathrm{Pt}(111)$. Indeed, the Co surface structure is known (a)

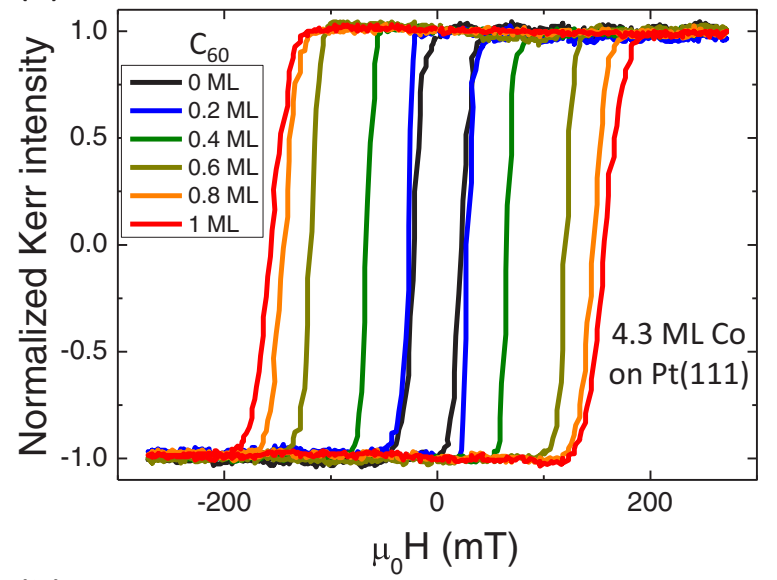

(b)

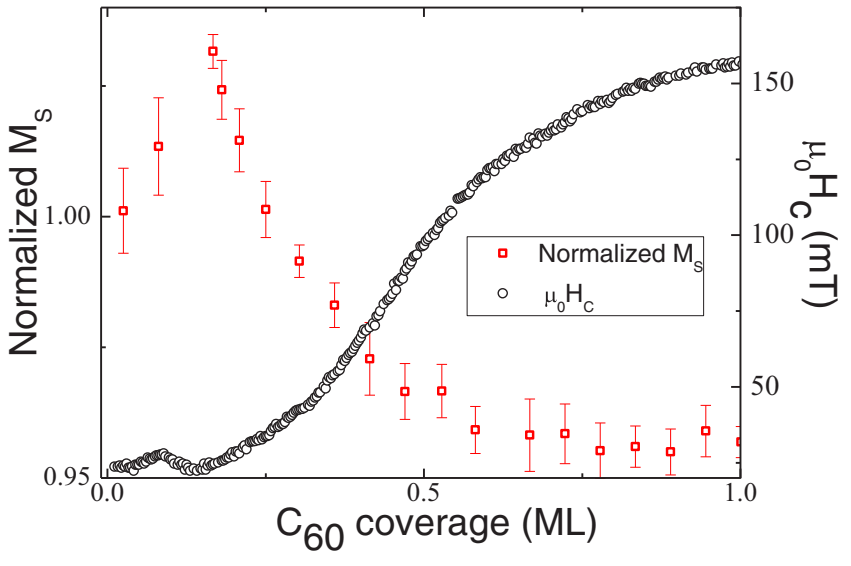

FIG. 2. (a) Raw hysteresis cycles (normalized to the same saturated intensity) recorded by polar MOKE in situ during the growth of $\mathrm{C}_{60}$ on 4.3 ML Co/Pt(111). Cycles are shown for each 0.2 ML increase of $\mathrm{C}_{60}$ coverage. (b) Variations of $\mu_{0} H_{c}$ and of the Kerr intensity at saturation (normalized to the one without $\mathrm{C}_{60}$ ) versus $\mathrm{C}_{60}$ coverage on 4.3 ML Co film on $\mathrm{Pt}(111)$.

to be different for ultrathin films on $\mathrm{Au}(111)$ and $\mathrm{Pt}(111)$. The SRT thickness of Co on $\mathrm{Pt}(111)$ has a large variety of values in literature [29]. Depending on the initial surface morphology of $\mathrm{Pt}(111)$, deposition method and rate, temperature, etc., it can vary from 4.4 ML [19] up to $12 \mathrm{ML}$ [29]. However, theoretical study predicts a SRT at around $4 \mathrm{ML}$ Co on Pt(111) [30]. In our case we observed a SRT thickness around 4.5 ML for Co on $\mathrm{Pt}(111)$. As we use the SRT for the calibration of the large Co thicknesses, we fix in the following this value of 4.5 ML, where the magnetization goes from out of plane to in plane.

To study the magnetic hardening at the $\mathrm{C}_{60} / \mathrm{Co}$ interface we have deposited $\mathrm{C}_{60}$ on an out-of-plane magnetized layer, namely 4.3 ML of Co on $\mathrm{Pt}(111)$. The evolution of the raw hysteresis cycles, as shown in Fig. 2(a), demonstrates clearly that the coercivity of the underlying Co film enhances with increasing $\mathrm{C}_{60}$ coverage. The coercive field $\left(H_{c}\right)$ enhances by a factor of almost 7 , where $\mu_{0} H_{c}$ value goes from 25 to $160 \mathrm{mT}$ after a full layer deposition of $\mathrm{C}_{60}$ as shown in Fig. 2(b). This finding of a strong hardening of a $\mathrm{C}_{60} / \mathrm{Co} / \mathrm{Pt}$ layer could be of particular interest for future spintronic devices with organic layers. It is worth noting that, in ultrathin films, the coercive 
field is far lower than the anisotropy field and is determined by the nucleation and propagation of magnetic domain walls. The so-called depinning field is proportional to the magnetic anisotropy but is also related to the strength of the defects that pin the magnetic domain wall during its propagation. Those defects are generally due to local variations of the domain wall energy, i.e., either of magnetic anisotropy or exchange. The local hybridization of molecules with an underlying ferromagnetic film can induce both those inhomogeneities of the anisotropy and exchange. This magnetic hardening of the Co layer on $\mathrm{Pt}(111)$ induced by a $\mathrm{C}_{60}$ deposition can therefore be interpreted by an increase of the magnetic anisotropy but not unambiguously. In Fig. 2(b) we also plot the Kerr intensity of the individual square cycles normalized by the intensity obtained on the bare Co film. It is worth noting that this variation of intensity can be due a priori in part to the optical absorption by the molecular layer but another part can reflect some change in the saturated magnetization of the Co layer. The fact that we observe a nonmonotonous variation with the $\mathrm{C}_{60}$ coverage strongly indicates that the main variation is indeed due to a change of magnetization. The overall decrease of a few percent for a full molecular coverage was also observed and calculated for other molecular layers [16,17,31] and is certainly due to the molecule cobalt hybridization. A new feature is the slight increase of $M_{S}$, observed together with a small decrease of the coercive field, in the very beginning of the $\mathrm{C}_{60}$ growth (below $0.2 \mathrm{ML}$ ).

As already observed for $\mathrm{Co}$ films on $\mathrm{Au}(111)$, the $\mathrm{C}_{60}$ overlayer can also induce a reverse spin reorientation transition from in-plane to out-of-plane magnetization. Figures 3(a) and 3(b) show two examples of such a process. In Fig. 3(a), for a Co thickness of 7.1 ML, the transition is already completed for 0.4 ML of $\mathrm{C}_{60}$. In Fig. 3(b), for 8.5 ML of Co, the transition is incomplete for a full coverage and open cycles are typical of a canted magnetization (note that the normalization to unity does not correspond in this case to the saturated magnetization and is set to an ad hoc value corresponding to the highest Kerr signal measured on this sample). However, the effect of the molecular coverage on the perpendicular magnetic anisotropy is rather impressive as it is able to stabilize out-of-plane magnetization for films almost twice thicker than without capping. Roughly, it means that the $\mathrm{Co} / \mathrm{C}_{60}$ interface has an interfacial magnetic anisotropy comparable to the $\mathrm{Pt} / \mathrm{Co}$ interface.

From a fundamental point of view, the comparison between the results obtained on Co films on $\mathrm{Pt}(111)$ and on $\mathrm{Au}(111)$ [16] is particularly interesting. For Co thickness below the SRT, the enhancement of the coervice field is by a factor 7 on $\mathrm{Co} / \mathrm{Pt}(111)$, whereas it is only by a factor of 2 on $\mathrm{Au}(111)$. For Co thickness above the SRT, the $\mathrm{C}_{60}$ capping on $\mathrm{Co} / \mathrm{Pt}(111)$ is able to maintain out-of-plane magnetization up to around $9 \mathrm{ML}$, whereas this is limited to approximately $6 \mathrm{ML}$ for $\mathrm{Co} / \mathrm{Au}(111)$. Those two experimental observations show that the interfacial magnetic anisotropy seems to be stronger for $\mathrm{Co} / \mathrm{C}_{60}$ on $\mathrm{Pt}(111)$ as compared to $\mathrm{Au}(111)$.

The main differences between few monolayer Co films grown on $\mathrm{Au}(111)$ and $\mathrm{Pt}(111)$ are the film roughness, the crystalline stacking, and the Co lattice parameters. Figures 4(a) and 4(b) show $50 \mathrm{~nm}$ wide STM images of $\mathrm{C}_{60}$ covered Co films on $\mathrm{Pt}(111)$ and $\mathrm{Au}(111)$, respectively. This mesoscopic scale allows one to observe at the same time the roughness (a)

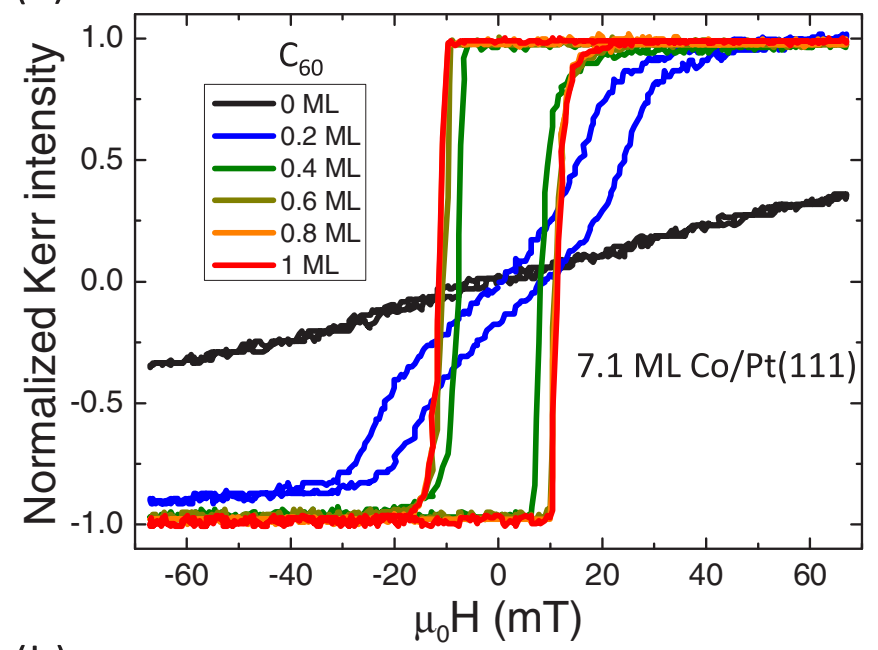

(b)

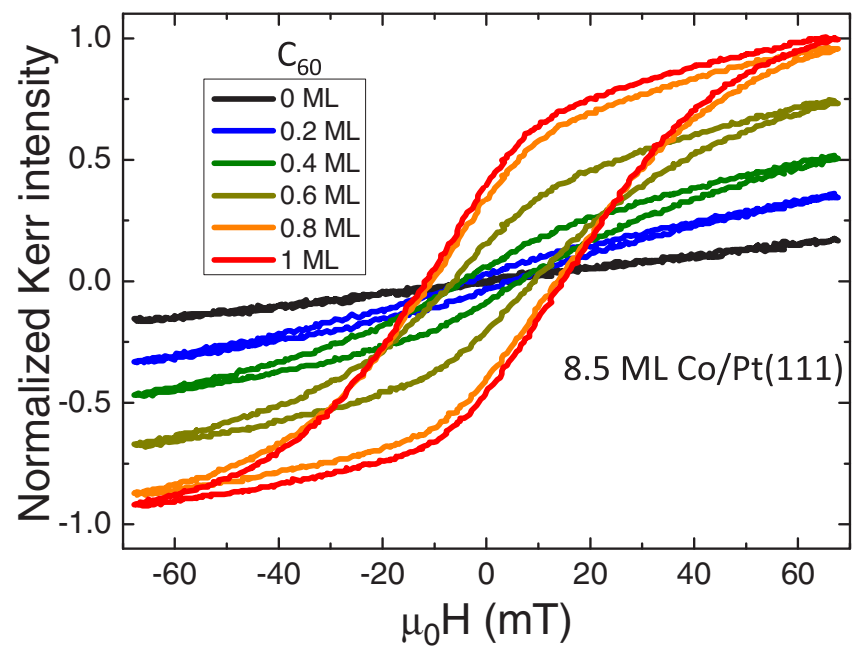

FIG. 3. (a) Raw hysteresis cycles (normalized to the saturated intensity) recorded by polar MOKE in situ during the growth of $\mathrm{C}_{60}$ on (a) 7.1 ML Co/Pt(111) and (b) 8.5 ML Co/Pt(111).

of the Co layer and the organization of the molecular layer. First, due to the nucleation of bilayer Co islands on $\mathrm{Au}(111)$ at the elbows of its herringbone surface reconstruction, the Co surface of ultrathin films on $\mathrm{Au}(111)$ shows a small scale roughness (terrace size limited to few nm) that is absent on $\mathrm{Co} / \mathrm{Pt}(111)$. This difference of morphology has an influence on the organization of $\mathrm{C}_{60}$. Indeed, a close look at Figs. 4(a) and 4(b) shows that the molecules are better crystallized on a hexagonal lattice $(4 \times 4$ as determined by low energy electron diffraction) on $\mathrm{Co} / \mathrm{Pt}(111)$, whereas on $\mathrm{Co} / \mathrm{Au}(111)$ only local order can be observed. The inset of Fig. 4(a) also shows that on the well-ordered area, we can observe that the favored $\mathrm{C}_{60}$ adsorption site is on a hexagon (threefold symmetry in the STM image). On $\mathrm{Co} / \mathrm{Au}(111)$, no preferential adsorption site could have been observed and the local change of size of $\mathrm{C}_{60}$ (due to electronic effects) is typical of random adsorption configurations. The influence of adsorption sites on the interfacial magnetic properties will be discussed in detail in the theoretical section. 
(a)

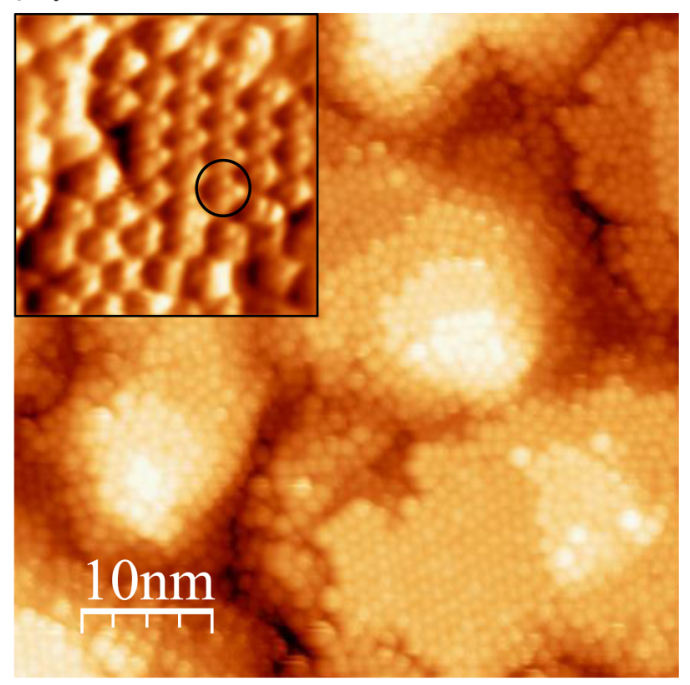

(b)

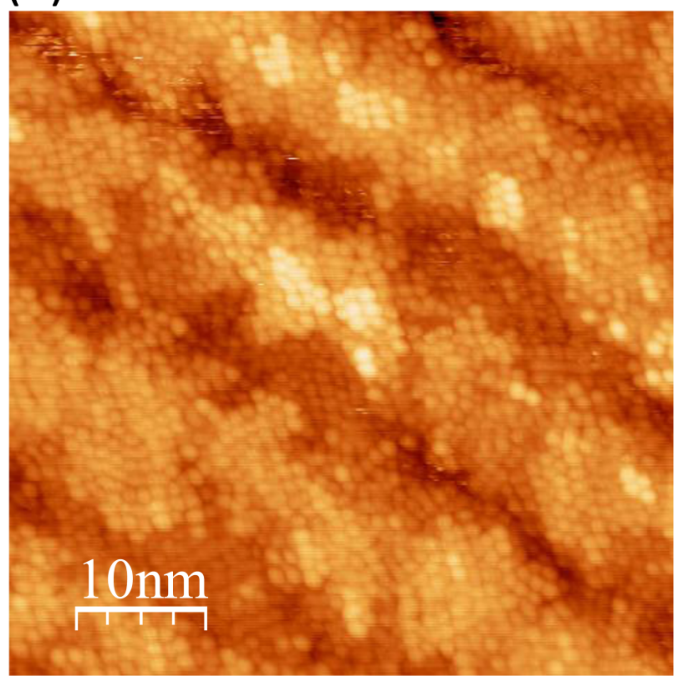

FIG. 4. $50 \times 50 \mathrm{~nm}^{2} \mathrm{STM}$ images of a full $\mathrm{C}_{60}$ layer (a) on $8 \mathrm{ML}$ Co film on $\mathrm{Pt}(111)$ ( $1 \mathrm{~V}, 0.3 \mathrm{nA}$, in inset, a $8 \times 8 \mathrm{~nm}^{2}$ current image showing the hexagonal adsorption configuration of $\mathrm{C}_{60}$ ) and (b) on 5.5 ML Co film on $\mathrm{Au}(111)$.

Another important difference between the two substrates is the Co stacking. On $\mathrm{Au}(111)$, the hexagonal stacking is clearly favored [32]. On $\mathrm{Pt}(111)$, the exact stacking is controversial. While a STM study has shown that the stacking is facecentered cubic for thickness above 8 ML [33], extended x-ray absorption fine structure spectroscopy has demonstrated that it is hexagonal close packed [34]. Finally, grazing incidence x-ray diffraction data show more quantitatively that the cobalt stacking on $\operatorname{Pt}(111)$ at room temperature for thickness around 4-6 ML is disordered, i.e., an equal probability to get locally a hexagonal close packed or a face centered cubic stacking [35]. The influence of the stacking (hcp of fcc) on the interfacial magnetic anisotropy will be discussed in the theoretical section.

Regarding strain, films on both substrates show an average in-plane lattice parameter close to the Co bulk one but (a)

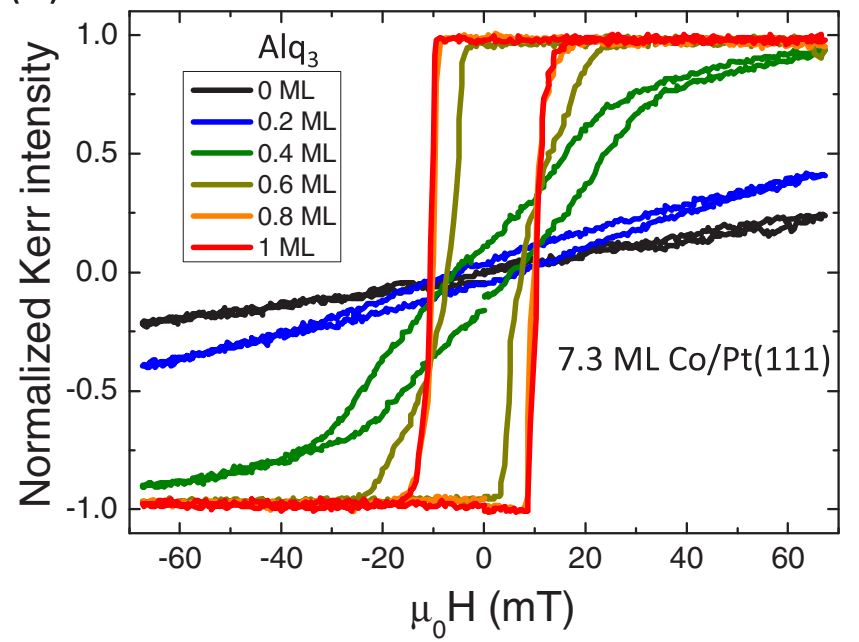

(b)

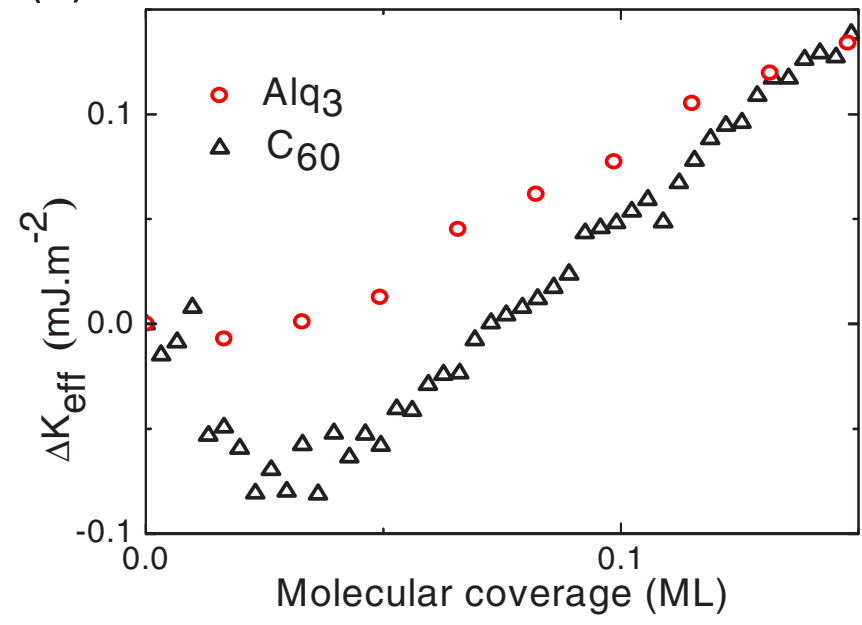

FIG. 5. (a) Raw hysteresis cycles (normalized to the saturated intensity) recorded by polar MOKE in situ during the growth of $\mathrm{Alq}_{3}$ on 7.3 ML Co/Pt(111). (b) Comparison of the change of interfacial magnetic anisotropy for $\mathrm{C}_{60}$ on $8.5 \mathrm{ML} \mathrm{Co} / \mathrm{Pt}(111)$ and $\mathrm{Alq}_{3}$ on 7.3 ML Co/Pt(111).

STM experiments [36] have revealed clear Moiré patterns on $\mathrm{Co} / \mathrm{Pt}(111)$ that are absent on $\mathrm{Co} / \mathrm{Au}(111)$. It is therefore very likely that the in-plane Co-Co distance is not homogeneous over the Co surface, at least for few monolayer Co films on $\mathrm{Pt}(111)$. This critical point will also be discussed in more detail in the theoretical section.

\section{C. $\mathbf{A l q}_{3} / \mathbf{C o} / \mathbf{P t}(\mathbf{1 1 1})$}

In order to have a more complete view of the PMA induced by a molecular overlayer, we have measured the magnetic properties of Co films on $\mathrm{Pt}(111)$ during the deposition of $\mathrm{Alq}_{3}$. Figure 5(a) shows that the effect is rather similar to that for a $\mathrm{C}_{60}$ overlayer. Using hard axis magnetometry when the magnetization is in plane, we can also deduce a more quantitative determination of the interfacial magnetic anisotropy and compare $\mathrm{Alq}_{3} / \mathrm{Co}$ and $\mathrm{C}_{60} / \mathrm{Co}$ interfaces. Figure 5(b) displays the variation with molecular coverage in the two cases, only for coverage below $0.15 \mathrm{ML}$, where the magnetization was 
fully in plane. The first observation is that the overall change of anisotropy is indeed stronger than for Co films on $\mathrm{Au}(111)$. Between 0.05 and $0.15 \mathrm{ML}$ of $\mathrm{C}_{60}$ coverage, the interfacial anisotropy increases by around $0.2 \mathrm{~mJ} \mathrm{~m}^{-2}$ on $\mathrm{Co} / \mathrm{Pt}(111)$, while it is only around $0.1 \mathrm{~mJ} \mathrm{~m}^{-2}$ for $\mathrm{Co} / \mathrm{Au}(111)$ [cf. Fig. 1(b)]. This larger value of the interfacial anisotropy for the $\mathrm{Co} / \mathrm{Pt}(111)$ system is consistent with the fact that we are able to stabilize out-of-plane magnetization for 8-9 ML Co films on $\mathrm{Pt}(111)$ with a $\mathrm{C}_{60}$ overlayer.

However, the two curves are rather different at low coverage, with a nonmonotonous behavior in the case of $\mathrm{C}_{60}$. The decrease of the magnetic anisotropy in the very beginning of the molecular growth can clearly be seen in raw cycles where the slope starts to decrease before increasing again and finally transforms into open cycles.

As a conclusion of this experimental section, the qualitative increase of perpendicular magnetic anisotropy due to a molecular overlayer on $\mathrm{Co}$ films is rather insensitive to the detail of the molecule $\left(\mathrm{C}_{60}\right.$ and $\left.\mathrm{Alq}_{3}\right)$ or the substrate [Au(111) and $\operatorname{Pt}(111)]$. However, quantitative differences are observed when comparing $\mathrm{C}_{60} / \mathrm{Co}$ on $\mathrm{Au}(111)$ and $\mathrm{Pt}(111)$. It makes $\mathrm{C}_{60} / \mathrm{Co} / \mathrm{Pt}$ a good candidate for high perpendicular magnetic anisotropy ultrathin films, with rather large coercive fields. From a more fundamental perspective, it shows that details of the interface matter, which has been recently pointed out for another important property of such interfaces, namely the induced spin polarization of $\mathrm{C}_{60}$ molecules adsorbed on different magnetic substrates [37]. In the following, we explore by means of tight-binding and density functional theory calculations the impact of different parameters like the Co stacking, the molecular adsorption geometry, and the strain in the Co layer on the induced interfacial magnetic anisotropy.

\section{THEORETICAL INVESTIGATIONS}

\section{A. Influence of the substrate on the Co layer}

In order to understand the influence of the substrate $\mathrm{Au}$ or $\mathrm{Pt}$ ) on the magnetic properties of the $\mathrm{C}_{60} / \mathrm{Co}$ interface we have performed a series of density functional theory and tight-binding calculations on systems as realistic as possible. One of the difficulties of modeling those systems is related to the large lattice mismatch between the Co layer and the Au or Pt substrate. Although the first neighbor distance $d_{\mathrm{Au}}\left(d_{\mathrm{Pt}}\right)$ is respectively $15 \%$ and $11 \%$ larger than $d_{\mathrm{Co}}$, the system adopts a configuration that allows a strain relaxation, as observed experimentally. If we note that $d_{\mathrm{Au}} / d_{\mathrm{Co}} \approx 8 / 7$ and $d_{\mathrm{Pt}} / d_{\mathrm{Co}} \approx$ $10 / 9$, an almost perfectly commensurate structure can been obtained since every eight (ten) Co atoms fall in almost perfect registry with every seven (nine) $\mathrm{Au}(\mathrm{Pt})$ atoms along a dense (first-neighbor) atomic row. Consequently, we have built up a plausible interface structure from an $8 \times 8(10 \times 10)$ supercell of Co hcp $(0001)$ in contact with a $7 \times 7(9 \times 9)$ supercell of $\mathrm{Au}(\mathrm{Pt}) \mathrm{fcc}(111)$. Nevertheless, the cobalt layer is still slightly stretched (compressed) when deposited on a gold (platinum) substrate (the gold and platinum in plane interatomic distance being fixed to their equilibrium value).

To obtain the atomic position of the substrate and Co thin film we have relaxed a system made of 20 layers of $\mathrm{Au}(\mathrm{Pt})$ and five layers of $\mathrm{Co}$, using a second moment approximation

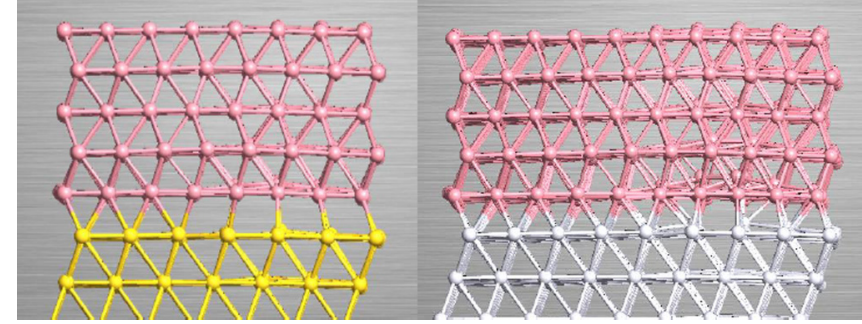

FIG. 6. Relaxed $\mathrm{Co} / \mathrm{Au}$ (left) and $\mathrm{Co} / \mathrm{Pt}$ (right) interfaces. The $\mathrm{Co} / \mathrm{Au}$ interface structure is built from an $8 \times 8$ supercell of $\mathrm{Co}$ hcp $(0001)$ in contact with a $7 \times 7$ supercell of $\mathrm{Au}$ fcc(111). The $\mathrm{Co} / \mathrm{Pt}$ interface structure is built from a $10 \times 10$ supercell of Co hcp $(0001)$ in contact with a $9 \times 9$ supercell of Pt fcc(111). We have adopted a finite slab geometry, i.e., with a limited number of atomic layers. Each atomic layer of the $\mathrm{Co} / \mathrm{Au}$ unit cell is made of 64 atoms of $\mathrm{Co}$ and 49 of $\mathrm{Au}$, while for the $\mathrm{Co} / \mathrm{Pt}$ unit cell it is made of 100 atoms of Co and 81 of $\mathrm{Pt}$.

potential [38]. The result is shown in Fig. 6. It clearly appears that the $\mathrm{Co} / \mathrm{Au}$ interface relaxes faster than the $\mathrm{Co} / \mathrm{Pt}$. In particular, the Co surface is almost "flat" when cobalt is grown on gold while a small atomic corrugation is still present when the substrate is platinum. In addition, due to the small remaining lattice mismatch of the superstructure the average interlayer distance of the cobalt film is slightly larger (by 1\%) when deposited on platinum than on gold. Finally, due to the corrugation observed at the surface of cobalt deposited on platinum (leading to a Moiré pattern) one can identify zones in slight compression and others in extension. To quantify this effect we have calculated for each surface atom the average in-plane first neighbor distance (over the six first neighbors). The resulting map is represented in Fig. 7 showing localized zones with $1.5 \%$ extensions surrounded by a zone with $1 \%$ compression.

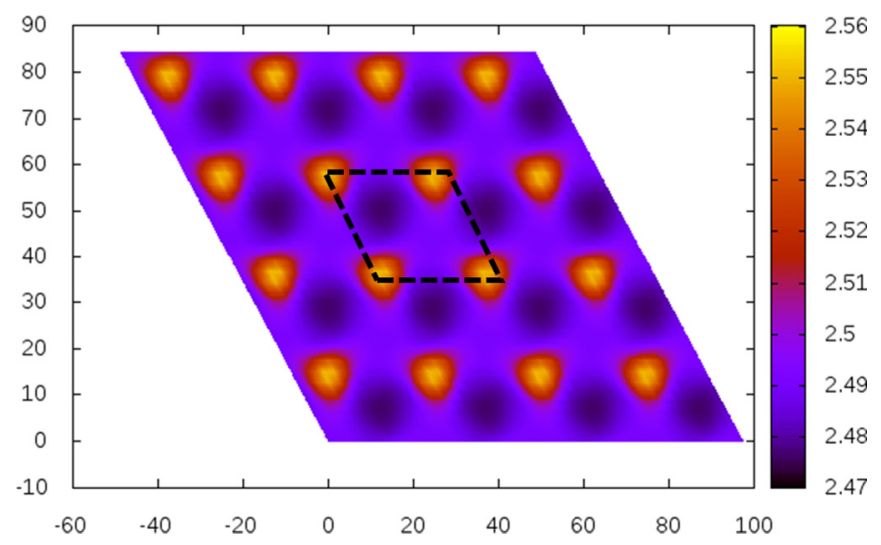

FIG. 7. Map of the average in-plane first neighbor distance (in angstrom) at the surface of cobalt when deposited on platinum. The corresponding structure is shown in Fig. 6 (right). Note that the equilibrium lattice parameter of bulk hcp cobalt is $a=2.51 \AA$. Yellow-orange regions are in extension, while dark blue regions are in compression. For better visualization we have represented a few unit cells. The elementary unit cell (containing $10 \times 10=100 \mathrm{Co}$ atoms) is indicated in dashed line. 


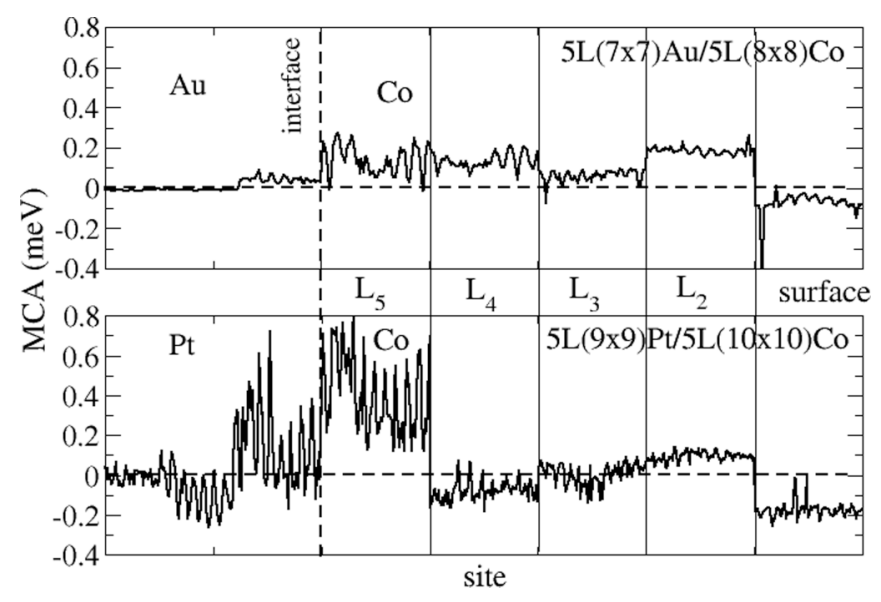

FIG. 8. Atom-resolved MCA of the two $\mathrm{Co} / \mathrm{Au}$ and $\mathrm{Co} / \mathrm{Pt}$ interfaces shown in Fig. 6. The dashed line shows the position of the $\mathrm{Co} / \mathrm{Au}(\mathrm{Co} / \mathrm{Pt})$ interface. The dotted lines separate the different $\mathrm{Co}$ layers, each containing 64 (100) atoms on $\mathrm{Au}(\mathrm{Pt})$.

The MCA is then evaluated within TB. Due to the size of the system the number of $k$ points has been restricted to $2 \times 2$ and $3 \times 3$ for calculations without and with SOC, respectively. In Fig. 8 we show the atomically decomposed MCA from the vicinity of the $(\mathrm{Au}$ and $\mathrm{Pt})$ interface to the cobalt surface. For both systems the MCA shows a very erratic behavior in the vicinity of the interface. The MCA of atoms belonging to the same atomic plane can change by more than $0.3 \mathrm{meV}$. In addition, the atoms from the substrate in direct contact with cobalt have a non-negligible contribution to the anisotropy and favor out-of-plane magnetization (MCA $>0$ ).

This is particularly pronounced in the case of platinum, although the layer beneath has the opposite tendency but smaller in amplitude (MCA $<0$ ). The MCA of the cobalt interface layer is also quite strongly reinforced compared to a regular cobalt slab without interface [23]. Finally, apart from the interface atoms one can note a global negative shift of the MCA in the cobalt layer when deposited on platinum rather than on gold. This can probably be attributed to the different stress since, as pointed out above, the interlayer distance of the cobalt film is slightly larger when deposited on platinum rather than on gold. To quantify this effect we have calculated the MCA of a bulk Co hcp as a function of the $a$ and $c$ parameters and verified that it is changing significantly. In particular the MCA decreases when $a$ decreases or when $c$ increases [39], which goes in line with a decrease of the MCA when the interlayer distance increases. All those results are in rather good agreement with the experimental findings with both positive interfacial anisotropies for the $\mathrm{Co} / \mathrm{Au}$ and $\mathrm{Co} / \mathrm{Pt}$ systems, with a stronger value for the $\mathrm{Co} / \mathrm{Pt}$ system. Adding the shape anisotropy, which has a negative value, leads to a total MAE that changes its sign with the Co thickness, displaying a spin reorientation transition from out-of-plane to in-plane magnetization, as observed experimentally.

Due to the extreme computational complexity of the full system where a $\mathrm{C}_{60}$ would be adsorbed on a supercell such as $(8 \times 8) \mathrm{Co} /(7 \times 7) \mathrm{Au}$ or $(10 \times 10) \mathrm{Co} /(9 \times 9) \mathrm{Pt}$, in the following we have adopted a simpler strategy that consists in ignoring the substrate but taking into account its possible influence.

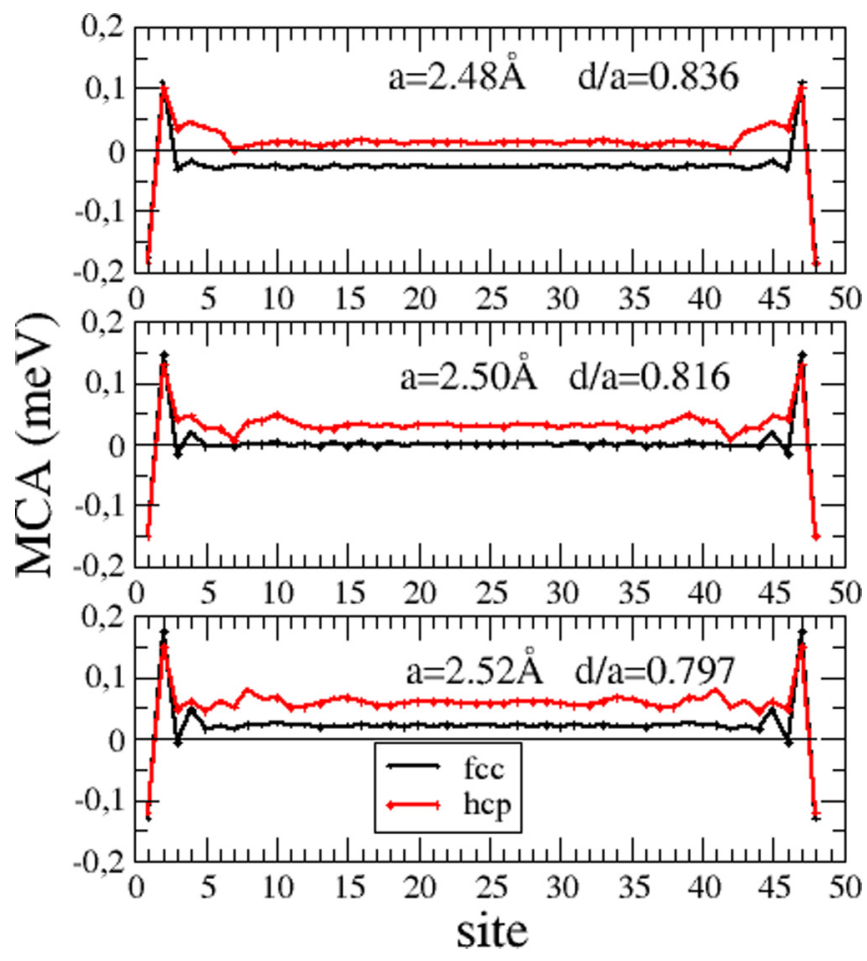

FIG. 9. Layer-resolved MCA of fcc(111) and hcp(0001) Co 48 layer slabs for three different in-plane lattice constants: $a=2.48 \AA$ (top), $a=2.50 \AA$ (middle), and $a=2.52 \AA$ (bottom). The interplane distance $d$ is determined to conserve the atomic volume $(d / a=$ $\frac{1}{2} \sqrt{8 / 3}$ for $a=2.50 \AA$ ).

There are basically three main influences of the substrate: (i) the stacking (hcp versus fcc; see Sec. IV B), (ii) the surface corrugation (larger in the case of Pt substrate), which could modify the adsorption geometry (see Sec. IVC), and (iii) the strain of the cobalt film (see Sec. IV D).

\section{B. Influence of the Co stacking: fcc versus hcp}

As previously mentioned the cobalt stacking on $\operatorname{Pt}(111)$ is a mixture of face centered cubic (fcc) and hexagonal closed packed (hcp), probably depending on the deposition conditions. In contrast on $\mathrm{Au}(111)$ the hcp stacking is favored. Therefore, in this section we will consider the influence of the stacking on the MCA.

Let us first consider slabs of Co in fcc(111) and hcp(0001) stacking. Since the cobalt layer is strained due to the lattice mismatch with its substrate we have also varied the in-plane lattice parameter (allowing compression and extension) and the interlayer distance keeping the atomic volume constant. The atom-resolved MCA shown in Fig. 9 is instructive and provides interesting information. First, as expected, the bulk MCA of the fcc stacking is zero for the in-plane lattice parameter $a=$ $2.50 \AA$ (corresponding to the equilibrium lattice constant) for which the bulk structure is perfectly cubic. In contrast, the bulk MCA of the hcp stacking is slightly positive $(0.035 \mathrm{meV} /$ atom $)$ favoring out-of-plane anisotropy. Slight variations of the inplane lattice constant have a non-negligible effect as already mentioned in the previous section: the MCA is increasing with $a$, while the difference between fcc and hcp remains 

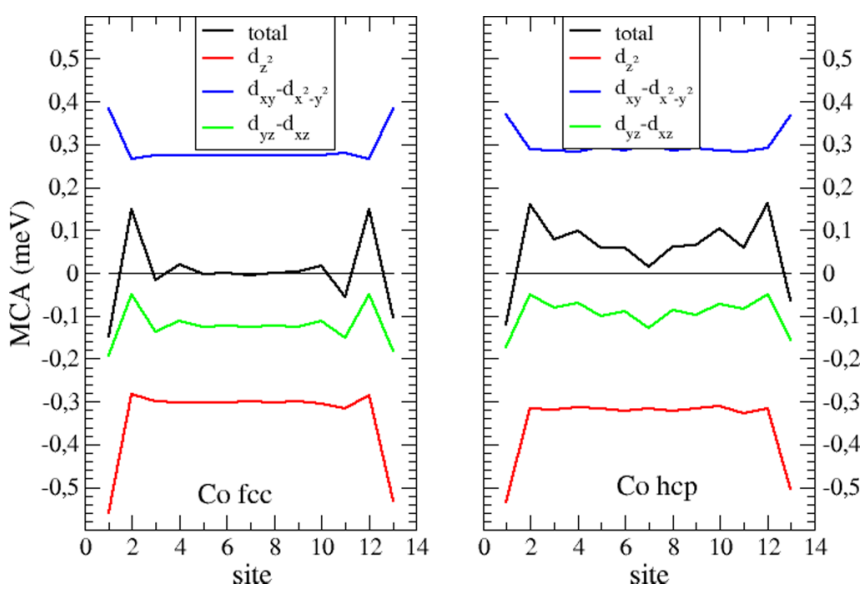

FIG. 10. Atom and orbital-resolved MCA of a 13 layer Co slab in fcc and hep stacking for an in-plane lattice parameter of $a=2.5 \AA$. $d_{z^{2}}$ is in red, $d_{x y}-d_{x^{2}-y^{2}}$ in blue, $d_{x z}-d_{y z}$ in green, and the total MCA in black. Only the atoms of "right" surface are allowed to relax.

almost constant. In the case of fcc stacking the MCA changes sign: favoring in-plane anisotropy for $a$ below $a=2.50 \AA$ and out-of-plane anisotropy for $a$ above $a=2.50 \AA$. The outermost atomic surface layer clearly favors in-plane anisotropy, while the sublayer favors out of plane in good agreement with our previous ab initio study [16]. Finally, it appears that, while the convergence of the MCA towards the bulk value is very fast for the fcc stacking, one observes long range oscillations for the hcp stacking. These types of oscillations are usually attributed to the existence of confined quantum well states between the two surfaces [40]. Note, however, that this oscillating behavior can probably only be observed in perfect systems. The presence of the $\mathrm{Au}(111)$ or $\mathrm{Pt}(111)$ substrates significantly perturbs the cobalt on several layers away from the interface and such oscillations will probably be washed out.

For further analysis it is interesting to perform an orbital decomposed MCA since the total MCA is a sum over the five $d$ orbitals. Due to the symmetry of the surface we have considered the contribution from $d_{z^{2}}, d_{x z}-d_{y z}$ and $d_{x y}-d_{x^{2}-y^{2}}$ (average values). From Fig. 10 it appears that the strong variation of MCA at the outermost layer is essentially related to the $d_{z^{2}}$ orbital that is almost constant (and negative) all over the slab except at the outermost layer where the MCA goes approximately from $-0.3 \mathrm{meV}$ to $-0.5 \mathrm{meV}$, strongly favoring in-plane anisotropy. The variation is slightly larger when Co is in fcc than in hcp stacking. The sublayer increase of the total MCA is due to the $d_{x z}-d_{y z}$ component. And the quantum well oscillation observed for the hcp stacking solely originates from $d_{x z}-d_{y z}$. Note also that in Fig. 10 the MCA curves are slightly asymmetric, i.e., the left and right surfaces are not exactly equivalent. This difference is attributed to the relaxation (with ATK software) that is allowed on the right surface only. The amplitude of the variation at the surface is slightly smaller on the relaxed surface.

Finally we have considered the case of a $\mathrm{C}_{60}$ molecule deposited on a Co slab in fcc and hcp stacking. The molecule is adsorbed in 5-6 bond geometry (see Sec. IVC for more details). Since, as we have shown in our previous ab initio study [16], the $d_{z^{2}}$ component of MCA $\left(\mathrm{MCA}_{z^{2}}\right)$ is the most affected
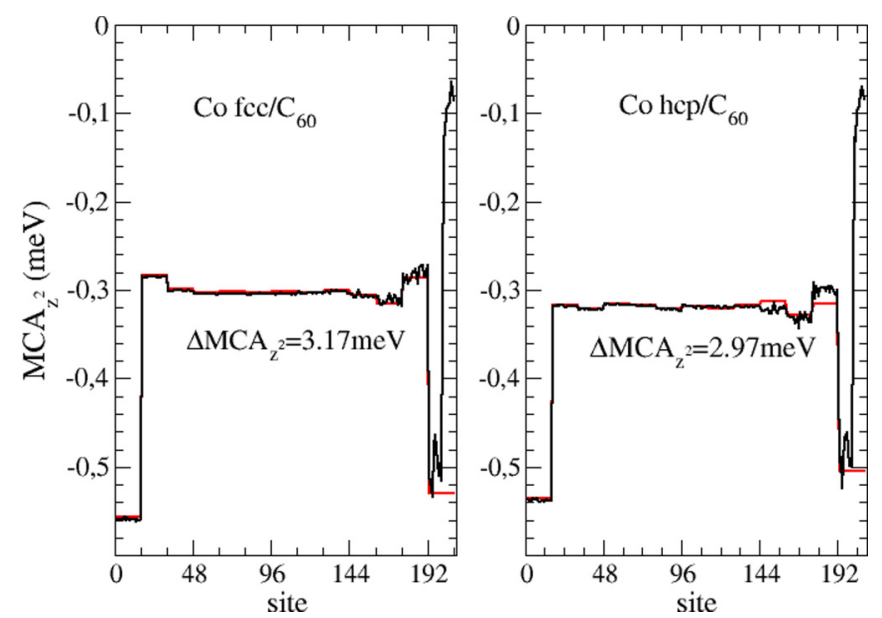

FIG. 11. Atom-resolved $d_{z^{2}}$ component of the MCA for a 13 layer slab of cobalt in fcc (left) and hcp (right) stacking. In black: surface in presence of $\mathrm{C}_{60}$. In red: bare Co surface.

by the adsorption of the molecule due to $p_{z}-d_{z^{2}}$ hybridization, we have calculated the layer-decomposed $\mathrm{MCA}_{z^{2}}$ for a slab in fcc and hcp stacking (see Fig. 11). We compare the bare Co surface, in red, with the Co surface with adsorbed $\mathrm{C}_{60}$, in black. Interestingly only the few surface atoms of cobalt that hybridize with carbon atoms of the buckyball (above site 200) are affected by the adsorption of the molecule. The $\mathrm{MCA}_{z^{2}}$ of those atoms is almost canceled. Integrating the difference between the Co slab with an adsorbed $\mathrm{C}_{60}$ and the bare surface we indeed find that the adsorption of the molecule favors an out-of-plane anisotropy since it cancels the in-plane component. The effect is slightly larger for an fcc stacking but is clearly not sufficient to explain the origin of the difference between $\mathrm{Co} / \mathrm{Au}(111)$ and $\mathrm{Co} / \mathrm{Pt}(111)$ films.

\section{Influence of the $\mathbf{C}_{60}$ adsorption geometry}

Let us now consider the influence of the adsorption geometry of a $\mathrm{C}_{60}$ molecule on the magnetism of the cobalt layer. To evaluate the influence of the adsorption geometry we have considered the case of a cobalt nine layer slab $(4 \times 4$ unit cell) on which is adsorbed a $\mathrm{C}_{60}$ molecule in three different geometries: a 5-6 bond, a hexagonal ring, and a pentagonal ring on top of a Co atom. The relaxed structures obtained via $\mathrm{QE}$ and ATK codes are very similar for the 5-6 bond and hexagonal geometry but for the pentagonal geometry one can note a stronger bonding in the case of ATK relaxation.

We have then performed tight-binding calculations including spin-orbit coupling. The atomically decomposed MCA for the six structures considered are shown in Fig. 12. We also show in red line the MCA for the bare Co slab, before the adsorption of $\mathrm{C}_{60}$. The variation of the total MCA upon adsorption of $\mathrm{C}_{60} \Delta \mathrm{MCA}=E_{\mathrm{C}_{60} / \mathrm{Co}}-E_{\mathrm{Co}}$ is very similar for QE and ATK 5-6 bond and hexagonal geometries, while for the ATK pentagonal geometry it is much higher than for the QE geometry. This can clearly be attributed to the stronger bonding observed for the ATK pentagonal geometry. Indeed the main effect of the $\mathrm{C}_{60}$ molecule on the MCA is essentially localized on the surface atoms that hybridize with the carbon atoms of the molecule. The total variation of MCA can change by a 

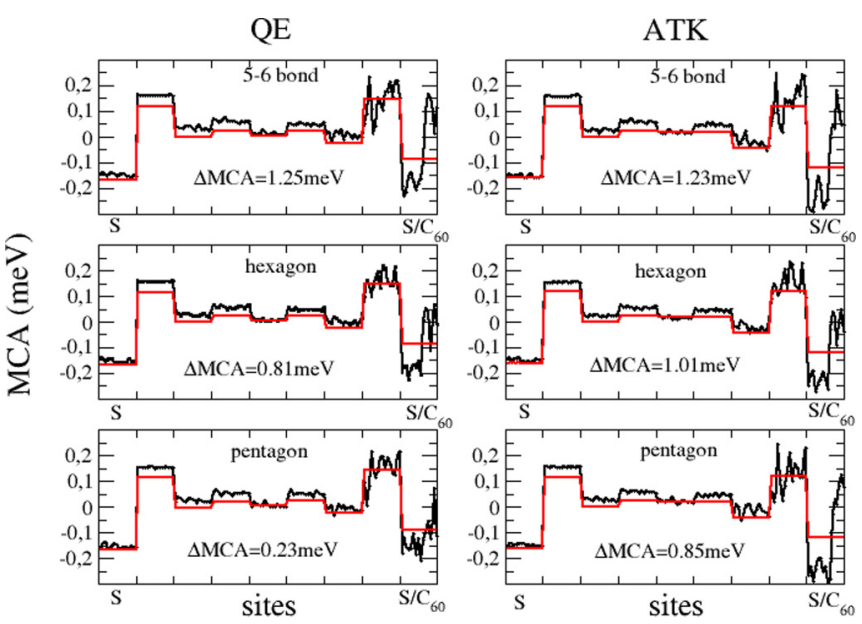

FIG. 12. Atom-resolved MCA of three different adsorption geometries of $\mathrm{C}_{60}$ on $\mathrm{Co}(0001)$ (5-6 bonds, hexagonal and pentagonal). The left graphs show the MCA for geometries relaxed with Quantum Espresso (QE) and the right graphs for geometries relaxed with Quantumwise (ATK). In red: bare Co surface. In black: surface in the presence of $\mathrm{C}_{60}$. The Co atoms hybridized with the molecule are the last atomic sites on the right of the graphs. The value of $\Delta \mathrm{MCA}=E_{\mathrm{C}_{60} / \mathrm{Co}}-E_{\mathrm{Co}}$ is indicated on each graph.

factor of five (0.23 for QE-pentagonal and 1.25 for 5-6 bond geometry). In particular, we observe that, in QE, the hexagonal adsorption site induces a magnetic anisotropy significantly higher than the pentagonal one. This could partly explain the higher magnetic anisotropy observed on the $\mathrm{Co} / \mathrm{Pt}$ system as compared to $\mathrm{Co} / \mathrm{Au}$, since hexagonal adsorption sites seem to be favored on $\mathrm{Co} / \mathrm{Pt}$, whereas random configurations are more likely on $\mathrm{Co} / \mathrm{Au}$ (cf. Fig. 4). It is worth noting that not only MCA but also local magnetic exchange could be modified in the Co layer due to the hybridization with $\mathrm{C}_{60}$ [41] and that it could also play a role in the magnetic hardening observed experimentally.

\section{Influence of the in-plane Co strain}

Finally, to investigate the influence of the strain of the cobalt film which is clearly different when Co is deposited on $\mathrm{Au}(111)$ (in-plane extension) or on $\mathrm{Pt}(111)$ (in-plane contraction) (see Figs. 6 and 7), we have considered a 19 layer $4 \times 4$ slab of cobalt with in-plane interatomic distance ranging from $2 \%$ contraction to $3 \%$ stretching. A $\mathrm{C}_{60}$ molecule in 5-6 bond geometry is then deposited on one side of the slab. To avoid any elastic or electronic interaction between the two surfaces of the slab a larger number of layers (19) has been used. The structures are relaxed using ATK and then the MCA is calculated via the tight-binding method. The results of our calculations show in Fig. 13 the atomically decomposed MCA over the whole slab for three different $\mathrm{Co}$ in-plane lattice parameters. We can note a clear upward shift (towards positive values) of the MCA when the in-plane interatomic distance is increased, which is in agreement with the magnetoelastic properties of Co. However, the shift is not rigid all over the slab: surface atoms are shifted differently from bulk ones. On the side where $\mathrm{C}_{60}$ is deposited the shape of the atomically decomposed MCA curve is hardly modified apart from a small shift but

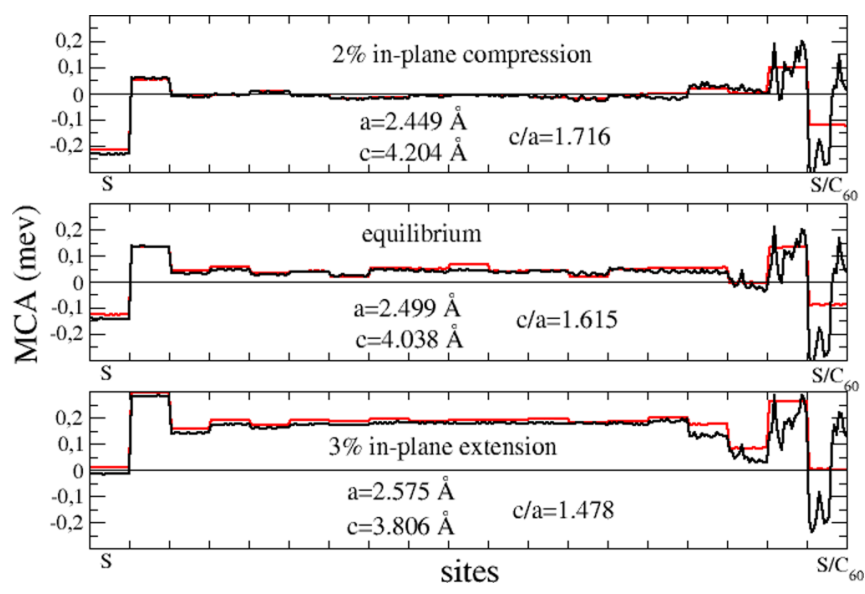

FIG. 13. Atom-resolved MCA of Co 19 layer slabs for various in-plane lattice parameters. In red: bare Co slab. In black: slab in presence of $\mathrm{C}_{60}$. The left part of the curve corresponds to the free surface (S) and the right part to the one in the presence of the buckyball $\left(\mathrm{C}_{60}\right) / \mathrm{S}$. The Co atoms hybridized with the molecule are the last atomic sites on the right of the graphs.

much less pronounced than the one of the bare surface (red). Finally, when integrating the difference (i.e., between bare surface and surface with $\mathrm{C}_{60}$ ) of MCA over the two outermost layers (32 atoms) a variation of more than $1.5 \mathrm{meV}$ is obtained between the stretched and the contracted slab. $\mathrm{C}_{60}$ deposited on a stretched region of the Co film favors out-of-plane anisotropy less than when deposited on a compressed region. This analysis shows that rather modest variations of surface strain can still have a non-negligible influence on the impact of the deposited molecule on the MCA of the magnetic layer. This is the most likely origin of the difference of behavior observed between $\mathrm{Co} / \mathrm{Au}(111)$ and $\mathrm{Co} / \mathrm{Pt}(111)$. Note that such strain induced effect on the interfacial magnetic anisotropy has been discussed very recently in $\mathrm{Co} / g r a p h e n e$ and $\mathrm{Co} / \mathrm{BN}$ heterostructures [42].

\section{CONCLUSION}

To conclude, we have shown that the induced perpendicular magnetic anisotropy in cobalt ultrathin films is observed for very different molecular overlayers like $\mathrm{Alq}_{3}$ and $\mathrm{C}_{60}$. We expect this effect to be general in the case of chemisorbed molecules on Co. However, an exhaustive study in the case of $\mathrm{C}_{60}$, both experimental and theoretical, demonstrates that the value of this interfacial anisotropy is very dependent on the details of the interface like the molecular adsorption geometry or the cobalt surface strain. We interpret those changes by different hybridization between the $\mathrm{C}_{60} \mathrm{p}_{z}$ and the Co $\mathrm{d}_{z^{2}}$ orbitals. A practical conclusion if one wants to use molecular overlayers to design magnetic anisotropy is that they need to control the fine structure of the interface, including the adsorption geometry of the molecules, which is certainly not an easy task in real devices.

\section{ACKNOWLEDGMENTS}

This project has received funding from the European Union's Horizon 2020 research and innovation programme under Grant No. 766726. 
[1] C. Barraud, P. Seneor, R. Mattana, S. Fusil, K. Bouzehouane, C. Deranlot, P. Graziosi, L. Hueso, I. Bergenti, V. Dediu et al., Nat. Phys. 6, 615 (2010).

[2] V. Dediu, L. E. Hueso, I. Bergenti, A. Riminucci, F. Borgatti, P. Graziosi, C. Newby, F. Casoli, M. P. De Jong, C. Taliani et al., Phys. Rev. B 78, 115203 (2008).

[3] D. Sun, L. Yin, C. Sun, H. Guo, Z. Gai, X.-G. Zhang, T. Z. Ward, Z. Cheng, and J. Shen, Phys. Rev. Lett. 104, 236602 (2010).

[4] M. Gobbi, F. Golmar, R. Llopis, F. Casanova, and L. E. Hueso, Adv. Mater. 23, 1609 (2011).

[5] R. Lin, F. Wang, M. Wohlgenannt, C. He, X. Zhai, and Y. Suzuki, Synth. Met. 161, 553 (2011).

[6] K. V. Raman, A. M. Kamerbeek, A. Mukherjee, N. Atodiresei, T. K. Sen, P. Lazic, V. Caciuc, R. Michel, D. Stalke, S. K. Mandal et al., Nature (London) 493, 509 (2013).

[7] Z. H. Xiong, D. Wu, Z. Valy Vardeny, and J. Shi, Nature (London) 427, 821 (2004).

[8] M. Cinchetti, V. A. Dediu, and L. E. Hueso, Nat. Mater. 16, 507 (2017).

[9] J. H. Shim, K. V. Raman, Y. J. Park, T. S. Santos, G. X. Miao, B. Satpati, and J. S. Moodera, Phys. Rev. Lett. 100, 226603 (2008).

[10] T. S. Santos, J. S. Lee, P. Migdal, I. C. Lekshmi, B. Satpati, and J. S. Moodera, Phys. Rev. Lett. 98, 016601 (2007).

[11] J. J. H. M. Schoonus, P. G. E. Lumens, W. Wagemans, J. T. Kohlhepp, P. A. Bobbert, H. J. M. Swagten, and B. Koopmans, Phys. Rev. Lett. 103, 146601 (2009).

[12] R. Lin, F. Wang, J. Rybicki, M. Wohlgenannt, and K. A. Hutchinson, Phys. Rev. B 81, 195214 (2010).

[13] Y.-J. Hsu, Y.-L. Lai, C.-H. Chen, Y.-C. Lin, H.-Y. Chien, J.-H. Wang, T.-N. Lam, Y.-L. Chan, D. H. Wei, H.-J. Lin et al., J. Phys. Chem. Lett. 4, 310 (2012).

[14] C. Vo-Van, Z. Kassir-Bodon, H. Yang, J. Coraux, J. Vogel, S. Pizzini, P. Bayle-Guillemaud, M. Chshiev, L. Ranno, V. Guisset et al., New J. Phys. 12, 103040 (2010).

[15] X. Zhang, S. Mizukami, T. Kubota, M. Oogane, H. Naganuma, Y. Ando, and T. Miyazaki, Appl. Phys. Lett. 99, 162509 (2011).

[16] K. Bairagi, A. Bellec, V. Repain, C. Chacon, Y. Girard, Y. Garreau, J. Lagoute, S. Rousset, R. Breitwieser, Y.-C. Hu et al., Phys. Rev. Lett. 114, 247203 (2015).

[17] M. Callsen, V. Caciuc, N. Kiselev, N. Atodiresei, and S. Blügel, Phys. Rev. Lett. 111, 106805 (2013).

[18] G. Rodary, V. Repain, R. L. Stamps, Y. Girard, S. Rohart, A. Tejeda, and S. Rousset, Phys. Rev. B 75, 184415 (2007).

[19] C. Shern, J. Tsay, H. Her, Y. Wu, and R. Chen, Surf. Sci. 429, L497 (1999).

[20] P. Giannozzi, S. Baroni, N. Bonini, M. Calandra, R. Car, C. Cavazzoni, D. Ceresoli, G. L. Chiarotti, M. Cococcioni, I. Dabo et al., J. Phys.: Condens. Matter 21, 395502 (2009).
[21] Atomistix ToolKit version 2015.1, QuantumWise A/S; www.quantumwise.com.

[22] M. Brandbyge, J.-L. Mozos, P. Ordejón, J. Taylor, and K. Stokbro, Phys. Rev. B 65, 165401 (2002).

[23] C. Barreteau, D. Spanjaard, and M.-C. Desjonquères, C. R. Phys. 17, 406 (2016).

[24] D. Li, A. Smogunov, C. Barreteau, F. Ducastelle, and D. Spanjaard, Phys. Rev. B 88, 219908(E) (2013).

[25] R. Allenspach, M. Stampanoni, and A. Bischof, Phys. Rev. Lett. 65, 3344 (1990).

[26] A. Caruso, D. Schulz, and P. Dowben, Chem. Phys. Lett. 413, $321(2005)$.

[27] Y.-P. Wang, X.-F. Han, Y.-N. Wu, and H.-P. Cheng, Phys. Rev. B 85, 144430 (2012).

[28] A. Droghetti, S. Steil, N. Großmann, N. Haag, H. Zhang, M. Willis, W.P. Gillin, A.J. Drew, M. Aeschlimann, S. Sanvito, and M. Cinchetti, Phys. Rev. B 89, 094412 (2014).

[29] J. Kim, J.-W. Lee, J.-R. Jeong, S.-C. Shin, Y. H. Ha, Y. Park, and D. W. Moon, Phys. Rev. B 65, 104428 (2002).

[30] U. Pustogowa, J. Zabloudil, C. Uiberacker, C. Blaas, P. Weinberger, L. Szunyogh, and C. Sommers, Phys. Rev. B 60, 414 (1999).

[31] P. Campiglio, R. Breitwieser, V. Repain, S. Guitteny, C. Chacon, A. Bellec, J. Lagoute, Y. Girard, S. Rousset, A. Sassella, M. Imam, and S. Narasimhan, New J. Phys. 17, 063022 (2015).

[32] Y. Girard, G. Baudot, V. Repain, S. Rohart, S. Rousset, A. Coati, and Y. Garreau, Phys. Rev. B 72, 155434 (2005).

[33] E. Lundgren, B. Stanka, M. Schmid, and P. Varga, Phys. Rev. B 62, 2843 (2000).

[34] J. Thiele, R. Belkhou, H. Bulou, O. Heckmann, H. Magnan, P. Le Fèvre, D. Chandesris, and C. Guillot, Surf. Sci. 384, 120 (1997).

[35] S. Ferrer, J. Alvarez, E. Lundgren, X. Torrelles, P. Fajardo, and F. Boscherini, Phys. Rev. B 56, 9848 (1997).

[36] P. Grüter and U. T. Dürig, Phys. Rev. B 49, 2021 (1994).

[37] D. Li, C. Barreteau, S. L. Kawahara, J. Lagoute, C. Chacon, Y. Girard, S. Rousset, V. Repain, and A. Smogunov, Phys. Rev. B 93, 085425 (2016).

[38] R. Cortes-Huerto, T. Sondon, and A. Saúl, J. Phys.: Condens. Matter 26, 474206 (2014).

[39] D. Sander, R. Skomski, A. Enders, C. Schmidthals, D. Reuter, and J. Kirschner, J. Phys. D 31, 663 (1998).

[40] J. Li, M. Przybylski, Y. He, and Y. Z. Wu, Phys. Rev. B 82, 214406 (2010).

[41] R. Friedrich, V. Caciuc, N. S. Kiselev, N. Atodiresei, and S. Blügel, Phys. Rev. B 91, 115432 (2015).

[42] B. S. Yang, J. Zhang, L. N. Jiang, W. Z. Chen, P. Tang, X.-G. Zhang, Y. Yan, and X. F. Han, Phys. Rev. B 95, 174424 (2017). 